\title{
Macro ingredientes como indicadores da qualidade de mistura de rações $^{1}$
}

\section{Macro ingredients as markers of the quality of mixing feed}

\author{
Diovani Paiano ${ }^{2 *}$; Héverton Michael Biazzi³; Cleisson Trevisan ${ }^{4}$; \\ Sidney Casarotto ${ }^{5}$; Fernando Zimmer ${ }^{6}$; Gustavo Krahl ${ }^{7}$; Leandro Sâmia Lopes ${ }^{2}$
}

\section{Resumo}

Foi conduzido um trabalho para avaliar o uso de macro ingredientes como indicadores da qualidade de mistura de rações. Foram utilizados (como marcadores): milho pipoca, sorgo, milheto, arroz colorido, milho roxo moído (adicionados em $0,5 \%$ na ração, em substituição ao milho moído) e o calcário grosso peneirado em peneira ABNT $\mathrm{n}^{\circ} 10$ com dois milímetros de diâmetro das malhas (adicionado em $0,5 \%$ na ração, em substituição ao calcário finamente moído). Como método referência utilizouse microtracer F-Red. Foi utilizado um misturador tipo Y (capacidade de $5.000 \mathrm{~cm}^{3}$ ) e fabricado $1 \mathrm{~kg}$ de ração para cada repetição. Foram utilizados seis tempos de mistura (10 segundos; $1 ; 6 ; 11 ; 16$ e 21 minutos) e cinco diâmetros geométricos médios do milho $(386 ; 513 ; 610 ; 731$ e $839 \mu \mathrm{m})$ totalizando 30 tratamentos, com três repetições por tratamento. Foram coletadas 12 amostras para a contagem dos indicadores e determinação do coeficiente de variação $(\mathrm{CV})$, para cada unidade experimental. Houve efeito quadrático com ponto de mínimo para os $\mathrm{CV}$ de todos os indicadores com o aumento do tempo de mistura. Não houve efeito do diâmetro geométrico médio do milho para os $\mathrm{CV}$ do calcário grosso e milheto. Houve efeito quadrático do diâmetro geométrico médio do milho para os $\mathrm{CV}$ do milho roxo e milho pipoca e efeito linear para o arroz colorido, sorgo e para o método referência. A granulometria do milho alterou o tempo necessário para reduzir o $\mathrm{CV}$ dos indicadores: arroz colorido, milho roxo moído, milho pipoca, sorgo e microtracer. A utilização de indicadores alternativos se mostrou promissora como ferramenta para determinação dos tempos ideais de mistura, com destaque para o milheto e o calcário, pois apresentaram as maiores correlações com o método referência.

Palavra-chave: Granulometria, homogeneidade de mistura, milho, tempo de mistura

\begin{abstract}
A study was conducted to evaluate the use of macro ingredients as markers of the quality of mixed feeds. Were used: popcorn grain, sorghum, millet, rice colored and purple corn grinded $(0.5 \%$ added to the diet, substituting grind corn) and coarse limestone sieved through sieve $\mathrm{n}^{\circ} 10$ ABNT with $2 \mathrm{~mm}$ (added in $0.5 \%$ in the diet, substituting finely ground limestone). Microtracer F-Red was used as a method of reference. It was used a Y-type mixer (capacity of 5,000 $\mathrm{cm}^{3}$ ), and produced $1 \mathrm{~kg}$ of feed per repetition.
\end{abstract}

\footnotetext{
${ }^{1}$ Parte do trabalho do segundo autor, PROBIC/UDESC, desenvolvido no Departamento de Zootecnia/DZO do Centro de Educação Superior do Oeste/CEO, Universidade do Estado de Santa Catarina, UDESC.

${ }^{2}$ Profs. do Dept ${ }^{\mathrm{o}}$ de Zootecnia, UDESC, Chapecó, SC. E-mail: diovani@hotmail.com; leandrosamia@uol.com.br

${ }^{3}$ Zootecnista, Poli-Nutri Nutrição Animal, Treze Tilia, SC. E-mail: heverton.biazzi@hotmail.com

${ }^{4}$ Zootecnista, Granja Colmeia Yagro, COLMEIAYAGRO, Uberlândia, MG. E-mail: cleissontrevisan@yahoo.com.br

${ }^{5}$ Zootecnista, Aurora Alimentos, Chapecó, SC. E-mail: sidney_casarotto@hotmail.com

${ }^{6}$ Zootecnista, Pesquisador Cooperativa Agroindustrial Alfa, ALFA, Chapecó, SC. E-mail: fernandozimmer2505@hotmail.com

${ }^{7}$ Zootecnista, Discente do Curso de Mestrado em Ciência Animal, Centro de Ciências Agroveterinárias, CAV/UDESC, Bolsista CAPES, Chapecó, SC. E-mail: gustavo.zootecnista@live.com

* Autor para correspondência
} 
It was adopted six mixing times (10 seconds, 1, 6, 11, 16 and 21 minutes) and five particle size of corn $(386,513,610,731$ and $839 \mu \mathrm{m})$, amounting 30 treatments with three repetitions for each treatment. Where collected 12 samples, for each replicate, to measurement of the markers and determination of the coefficient of variation (CV). A quadratic effect with minimum point for the $\mathrm{CV}$ was observed for all markers with the increasing of the mixing time. There was no effect of the particle size of corn for the coefficients of variation of coarse limestone and millet. There was a quadratic response of the particle size of corn for the coefficients of variation of purple corn and popcorn grain and linear effect for the colored rice, sorghum and reference method. The particle size of corn changed the time needed to reduce the $\mathrm{CV}$ of the markers: colored rice, milled purple corn, popcorn, sorghum and microtracer. The use of alternative markers has shown that it is a promising tool for the determination of the optimum mixing time, especially millet and limestone, that showed the highest correlations with the reference method.

Key words: Corn, mixing time, particle size, uniformity of mixing feed

\section{Introdução}

A produção de rações no Brasil em 2012 consumiu cerca de 65 milhões de toneladas de ingredientes e a previsão para 2013 é um consumo de cerca de 67 milhões de toneladas (ZANI, 2013), valores que indicam a grande relevância do setor.

Para a correta elaboração de rações e o aproveitamento do máximo potencial nutritivo dos alimentos, e consequente melhoria no desempenho e viabilidade econômica dos sistemas produtivos, é fundamental o controle dos pontos críticos da produção, os quais vão do recebimento à expedição dos produtos, com destaque à correta mistura dos ingredientes, etapa realizada com o intuito de dispersar o mais uniformemente possível as matérias-primas no produto final.

Uma mistura adequada permitirá fornecer ao animal uma dieta balanceada, por consequência, favorecer o consumo regular de nutrientes e maximizar o desempenho animal (McCOY et al., 1994). Segundo Mello, Pupa e Hannas (2003), rações mal misturadas causam problemas de desuniformidade dos lotes, canibalismo, baixas taxas nos índices reprodutivos, maior susceptibilidade as doenças, baixa imunidade e consumo irregular de aditivos quando utilizados.

As indústrias aceitam como parâmetro técnico de dispersão dos ingredientes um coeficiente de variação $(\mathrm{CV})$ igual ou menor a 10\% (JOHNSTON; SOUTHERN, 2000). Porém, para frangos de corte, McCoy et al. (1994), obtiveram máximo desempenho com o CV da ração entre 12 e $23 \%$, dependendo do método da análise utilizado. Em estudo similar com leitões Groesbeck et al. (2007) relataram melhora linear para o consumo, ganho de peso e conversão alimentar com a redução do $\mathrm{CV}$ das rações de $15,2 \%$ para 3,3\%. Da mesma forma Traylor et al. (1994) observaram piora no ganho de peso e na conversão alimentar de suínos, com o aumento do $\mathrm{CV}$ das rações.

$\mathrm{Na}$ prática, podem ser utilizados diversos elementos como indicadores para a avaliação da dispersão dos ingredientes na ração e consequentemente da qualidade da mistura. Clark, Behnke e Poole (2007), estudaram a DLMetionina, L-Lisina-HCl, proteína bruta, cloreto de sódio, manganês, partículas de ferro coloridas (microtracers) e antimicrobianos da dieta, sendo que dos diferentes indicadores estudados somente a proteína bruta e o cloreto de sódio não apresentaram redução no $\mathrm{CV}$ com o maior tempo da mistura. Segundo os autores os elementos da ração como a proteína e/ou o fósforo, por serem nutrientes provenientes da combinação de diversos ingredientes, como o milho, farelo de soja, entre outros, podem não ser bons indicativos da qualidade da mistura, e sugerem avaliar ingredientes de baixa inclusão, como aminoácidos ou antibióticos.

Entretanto, a quantificação dos indicadores citados pode encarecer a análise e aumentar o tempo para a sua quantificação e avaliação de sua 
dispersão na ração, o que dificulta a rápida correção de problemas de mistura.

Somado a estes fatores, deve ser destacado que ingredientes ou indicadores com densidade e granulometria excessivamente diferente aos outros componentes da ração podem causar possíveis erros na avaliação do CV da mistura por sua dispersão não ser homogênea (AXE, 1995).

Neste sentido, a utilização de ingredientes de fácil e rápida identificação, com características semelhantes aos ingredientes comumente utilizados nas rações, pode ser uma alternativa para a avaliação da qualidade de mistura de rações de forma rápida, como metodologia complementar aos métodos convencionais. Somado aos fatos citados destacase a escassa produção bibliográfica na literatura especializada tratando do assunto.

Diante do exposto, foi realizado este trabalho com o objetivo de avaliar a viabilidade da utilização de macro ingredientes (milho roxo moído, milho pipoca, sorgo, arroz, milheto e calcário) como indicadores para a determinação da qualidade da mistura e tempo ideal de funcionamento de misturadores.

\section{Material e Métodos}

O experimento foi conduzido no Laboratório de Nutrição Animal do Departamento de Zootecnia da Universidade do Estado de Santa Catarina. Foram utilizados seis ingredientes como indicadores alternativos, denominados de macro-ingredientes, milho pipoca, sorgo, milheto, arroz colorido, milho roxo moído (adicionados em 0,5\% na ração, em substituição ao milho moído) e calcário grosso, peneirado em peneira com malha de dois milímetros de diâmetro (adicionado em $0,5 \%$ na ração, em substituição ao calcário finamente moído). Paralelamente, foi utilizado o microtracer F-red com inclusão de $75 \mathrm{~g} /$ tonelada (aproximadamente 1000 unidades por $\mathrm{kg}$ de ração) o qual foi considerado como método referência para a determinação da homogeneidade da ração.

Foi utilizado como modelo para o estudo uma ração de custo mínimo, calculada para atender as exigências nutricionais de poedeiras semi-pesadas entre a $13^{\mathrm{a}}$ e $18^{\mathrm{a}}$ semana, conforme recomendações de exigências nutricionais e de composição nutricional de alimentos propostas por Rostagno et al. (2011).

As rações foram fabricadas em um misturador de aço inox, tipo $Y$, com capacidade da câmara de mistura de $5.000 \mathrm{~cm}^{3}$ com 24 rotações/minuto, com a seguinte sequência de abastecimento do misturador: milho $(732,45 \mathrm{~g})$, Indicadores $(5,00 \mathrm{~g}$ cada), prémistura de vitaminas e minerais $(3,00 \mathrm{~g})$, metionina $(0,07 \mathrm{~g})$, sal comum $(2,92 \mathrm{~g})$, calcário fino $(6,82 \mathrm{~g})$, fosfato bicálcico $(10,87 \mathrm{~g})$, farelo de soja $(136,81 \mathrm{~g})$ e farelo de trigo $(77,06 \mathrm{~g})$, totalizando $1 \mathrm{~kg}$ de ração.

Os CV dos indicadores foram avaliados em seis diferentes tempos de mistura (10 segundos; $1 ; 6 ; 11 ; 16$ e 21 minutos) e em cinco diferentes diâmetros geométricos médios (DGM) para o milho totalizando 30 tratamentos (Tabela 1).

Tabela 1. Características físicas do milho utilizado após as diferentes moagens.

\begin{tabular}{|c|c|c|c|c|c|}
\hline \multicolumn{6}{|c|}{ Características físicas das frações de milhos utilizados } \\
\hline Diâmetro geométrico médio, $\mu \mathrm{m}$ & 385 & 513 & 610 & 731 & 839 \\
\hline Desvio padrão geométrico & 1,42 & 1,80 & 1,66 & 1,94 & 2,38 \\
\hline Ângulo de repouso, ${ }^{\circ}$ & 26,89 & 19,62 & 22,22 & 19,08 & 18,55 \\
\hline Matéria seca, $\%$ & 86,80 & 86,54 & 87,63 & 86,87 & 87,19 \\
\hline Densidade, $\mathrm{g} / \mathrm{cm}^{3}$ & 0,526 & 0,576 & 0,598 & 0,606 & 0,736 \\
\hline
\end{tabular}

Fonte: Elaboração dos autores. 
Para a obtenção das diferentes granulometrias avaliadas, foi utilizado o mesmo lote de milho, subdividido em diferentes frações. Cada fração foi moída em diferentes peneiras e foram selecionadas as moagens que apresentavam cerca de $100 \mu \mathrm{m}$ de diferença no DGM do milho. Posteriormente foram avaliadas as características físicas e de matéria seca (Tabela 1). O farelo de soja e o farelo de trigo

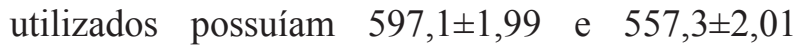
$\mu \mathrm{m}$ de DGM e desvio padrão geométrico (DPG), respectivamente, os quais foram obtidos no comércio local.

Após o abastecimento do misturador o equipamento foi acionado conforme os tempos pré-estabelecidos. Em cada unidade experimental foram coletadas 12 amostras com aproximadamente 65 gramas, por meio de um quarteador tipo "jones" de aço inox de 16 canais.

Nas amostras, os microtracer F-Red foram separados por meio de jarro de mason, conforme metodologia do fabricante (MICROTRACERS, 1999) e os demais indicadores quantificados. Com os valores obtidos para os indicadores para cada unidade experimental, foram calculados os $\mathrm{CV}$, conforme metodologia descrita por ASAE (1998) e Groesbeck et al. (2007).

Para determinação das características físicas dos ingredientes e macro ingredientes utilizados como indicadores (Tabelas 1 e 2) foram realizadas avaliações de: ângulo de repouso, diâmetro, esfericidade (MAGALHÃES et al., 2000), densidade (MOREIRA; CHAVES; OLIVEIRA, 1985), peso de mil unidades, DGM e DPG dos ingredientes (HANDERSON; PERRY, 1955).

Tabela 2. Características dos macro-ingredientes utilizados como indicadores.

\begin{tabular}{lllllll}
\hline \multirow{2}{*}{ Características } & \multicolumn{7}{c}{ Indicadores } \\
\cline { 2 - 7 } & $\begin{array}{l}\text { Arroz } \\
\text { colorido }\end{array}$ & $\begin{array}{l}\text { Calcário } \\
\text { grosso }\end{array}$ & Milheto & $\begin{array}{l}\text { Milho } \\
\text { Roxo }\end{array}$ & $\begin{array}{l}\text { Milho } \\
\text { Pipoca }\end{array}$ & Sorgo \\
\hline Peso de mil unidades, g & 19,80 & 4,97 & 4,92 & 19,67 & 56,39 & 36,13 \\
Desvio padrão do peso & 0,04 & 0,05 & 0,03 & 0,29 & 0,13 & 0,08 \\
Densidade, $\mathrm{g} / \mathrm{cm}^{3}$ & 0,81 & 1,41 & 0,80 & 0,49 & 0,78 & 0,80 \\
Esfericidade, \% $^{3}$ & 43,90 & 58,39 & 74,68 & 61,56 & 69,51 & 78,21 \\
Diâmetro, mm & 2,72 & 0,86 & 1,04 & 4,23 & 8,57 & 5,65 \\
Matéria seca, \% $^{\text {Ângulo de repouso, }}{ }^{\circ}$ & 88,07 & 99,93 & 88,70 & 84,58 & 86,26 & 87,53 \\
$\mathrm{~N}^{\mathbf{0}}$ de indicadores/kg de ração & 5,18 & 12,49 & 6,20 & 15,99 & 4,32 & 9,73 \\
\hline
\end{tabular}

Fonte: Elaboração dos autores.

Foi utilizado um delineamento inteiramente ao acaso com 30 tratamentos em um esquema fatorial $6 \times 5$ (seis tempos de mistura e cinco granulometrias) com três repetições, com um total de 90 unidades experimentais. Foram ajustados modelos de superfície de resposta, com o seguinte modelo estatístico:

$\mathrm{Y}_{\mathrm{ijk}}=\mu+\mathrm{T}_{\mathrm{i}}+\mathrm{G}_{\mathrm{j}}+\left(\mathrm{T}^{*} \mathrm{G}\right)_{\mathrm{ij}}+\mathrm{e}_{\mathrm{ijk}}$, em que: $\mathrm{Y}_{\mathrm{ijk}}=$ valor observado do marcador $\mathrm{k}$, do tempo i e do diâmetro $\mathrm{j} ; \mu=$ constante geral; $\mathrm{T}_{\mathrm{i}}=$ efeito do tempo de mistura i (10 segundos; $1 ; 6 ; 11 ; 16$ e 21 minutos); $\mathrm{G}_{\mathrm{j}}=$ efeito da granulometria do milho $\mathrm{j}(386 ; 513$; $610 ; 731$ e $839 \mu \mathrm{m}) ; \mathrm{T}^{*} \mathrm{G}_{\mathrm{ij}}=$ efeito da interação do tempo de mistura $\mathrm{i}$ com a granulometria $\mathrm{j}$ e $\mathrm{e}_{\mathrm{ijk}}=$ erro aleatório associado a cada observação.

Foram considerados para a elaboração das equações de superfície os modelos que apresentaram significância menor ou igual a $5 \%$, no caso de 
indicadores que apresentaram interação entre tempo de mistura e granulometria do milho, foram mantidos os polinômios de primeiro grau. No caso de indicadores que não apresentaram interação ou efeito da variável granulometria, foram avaliados considerando um modelo de regressão polinomial até a segunda ordem.

Para as variáveis que apresentaram efeito foram feitas as derivações das equações, para obtenção do tempo e do DGM que minimizassem os CV da mistura. Posteriormente, foram calculadas as correlações de Pearson entre os indicadores $(\mathrm{P}<0,01)$.

\section{Resultados e Discussão}

Foram observadas respostas diferentes para a análise dos $\mathrm{CV}$ dos diferentes indicadores (Tabela 3), o que sugere diferentes comportamentos de mistura dentro de cada granulometria e tempo para cada indicador, resultado de suas diferentes características como densidade, esfericidade, diâmetro entre outros. Possivelmente, esta característica é similar para os demais componentes da ração, com isso, há um tempo ideal de mistura específico para cada ingrediente e sugere que o tempo necessário de mistura para um ingrediente pode ser excessivo para outro componente da ração.

Tabela 3. Média dos coeficientes de variação de Indicadores em função do tempo de mistura e do diâmetro geométrico médio do milho.

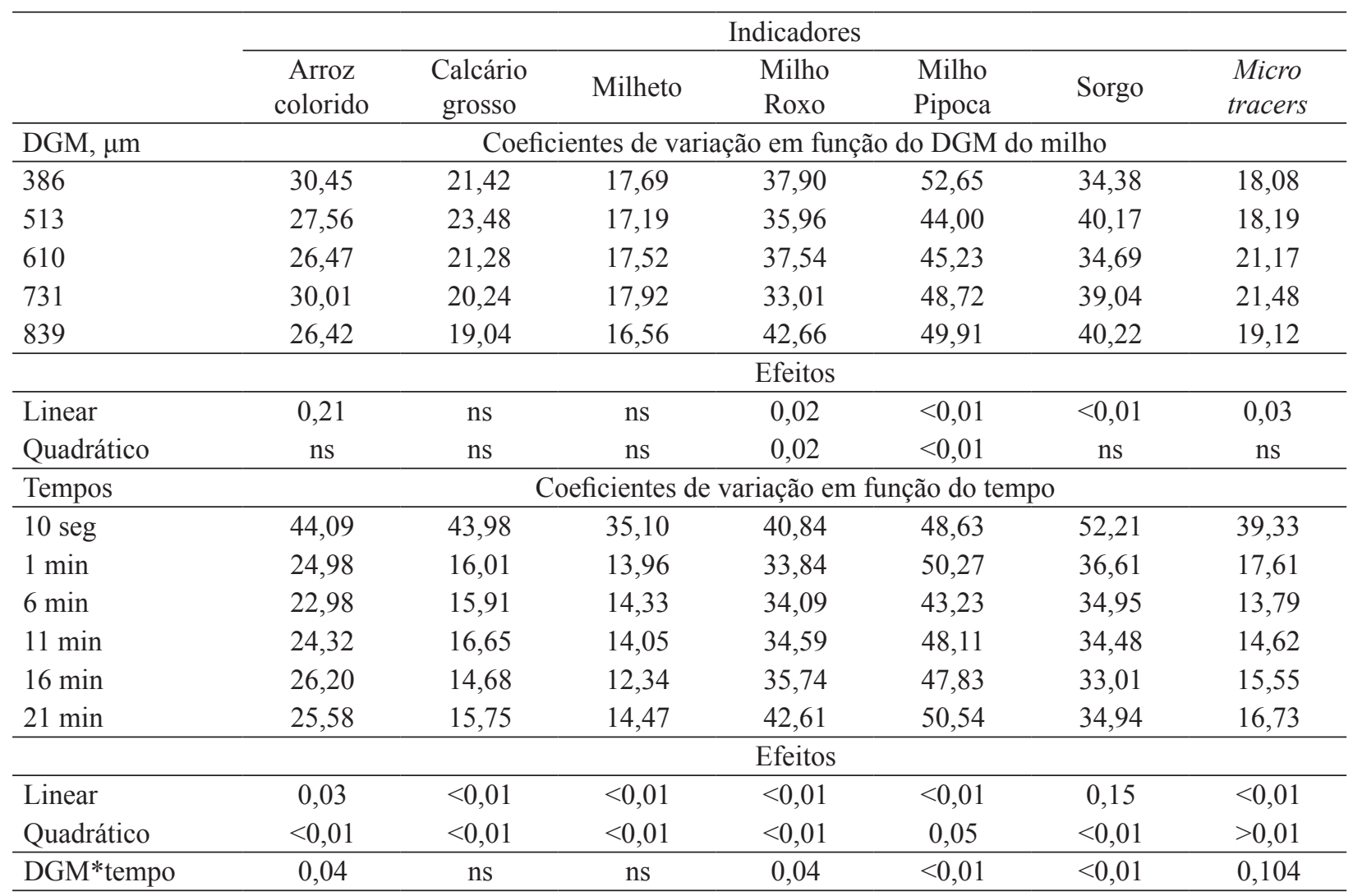

Fonte: Elaboração dos autores.

Foram observados efeitos quadráticos $(\mathrm{P} \leq 0,05)$, indicadores avaliados (Tabela 3$)$ com o aumento com ponto de mínimo, para todos os $\mathrm{CV}$ dos nos tempos de mistura. Estes resultados foram 
semelhantes aos obtidos por Clark, Behnke e Poole (2007), que verificaram efeito quadrático do tempo de mistura para os $\mathrm{CV}$ dos indicadores microtracers, L-lisina, $\mathrm{P}, \mathrm{Mn}$ e antimicrobiano e dos resultados de McCoy et al. (1994) os quais observaram efeito quadrático do tempo de mistura para os CV do sal e microtracers em rações para frangos de corte. Provavelmente, o resultado obtido seja consequência de fatores como cargas elétricas e características físicas dos ingredientes, que levaram à desmistura das rações nos tempos excessivos de funcionamento do misturador. Além do processo de desmistura, com consequente prejuízo ao desempenho zootécnico, o tempo excessivo de funcionamento promove maior gasto em energia elétrica e redução da vida útil do equipamento.

Outro fator a ser considerado é o modelo matemático utilizado, no qual foram testadas equações polinomiais até a $2^{\mathrm{a}}$ ordem, eventualmente outros modelos matemáticos, quando correlacionados como o desempenho animal, poderiam ser utilizados para a definição dos tempos ideais de mistura.

Houve interação $(\mathrm{P}<0,05)$ entre o DGM do milho e tempo de mistura para os $\mathrm{CV}$ dos indicadores: milho roxo moído, milho pipoca, arroz, sorgo, e para microtracer F-Red (Tabela 3).

Os CV das partículas de milho roxo moído e milho pipoca apresentaram efeito quadrático com ponto de mínimo $(\mathrm{P}<0,05)$ para diferentes granulometrias utilizadas e para os diferentes tempos de mistura $(\mathrm{P} \leq 0,05)$. Os pontos de inflexão foram obtidos com 9,34 minutos de mistura e 616,4 $\mu \mathrm{m}$ de DGM para o milho pipoca e 9,37 minutos de mistura e $577,5 \mu \mathrm{m}$ de DGM para o milho roxo moído, respectivamente (Figura 1). Possivelmente, o milho pipoca pelo seu grande diâmetro $(>8 \mathrm{~mm})$ e o milho roxo moído pela sua baixa densidade $\left(0,49 \mathrm{~g} / \mathrm{cm}^{3}\right)$, em relação aos demais indicadores, tenham contribuído para tais resultados.

Figura 1. Superfícies de respostas dos coeficientes de variação do milho pipoca e milho roxo moído em rações fabricadas com diferentes tempos de mistura e diâmetros geométricos do milho.
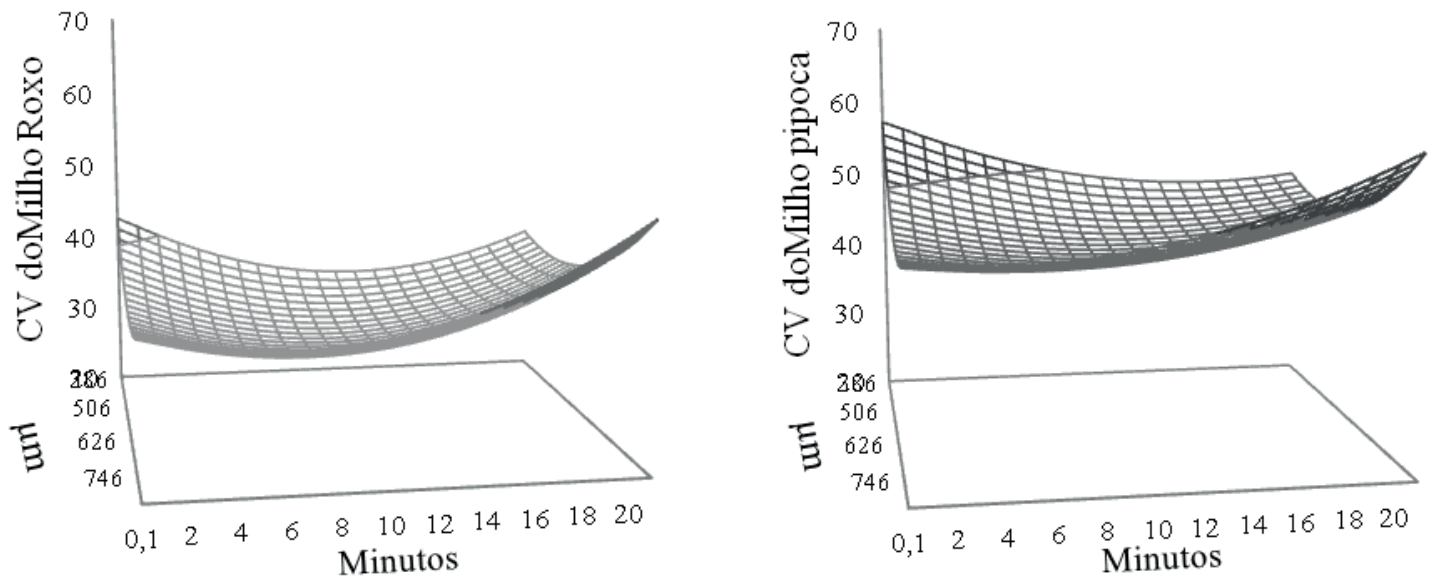

Milho pipoca: $\mathrm{CV}=105,9520-2,02526 \mathrm{~T}+0,0373970 \mathrm{~T}^{2}-0,1743190 \mathrm{D}+0,0001251 \mathrm{D}^{2}+0,0021520 \mathrm{TD}$ Milho roxo moído: $C V=80,2921-2,07953 \mathrm{~T}+0,0613244 \mathrm{~T}^{2}-0,1385420 \mathrm{D}+0,0001069 \mathrm{D}^{2}+0,0016114 \mathrm{TD}$.

Fonte: Elaboração dos autores.

Deve ser destacado que o milho roxo moído e o milho pipoca são os indicadores com a maior similaridade (Tabelas 1 e 2) com o milho utilizado nas rações. Assim, os efeitos quadráticos com ponto de mínimo para as diferentes granulometrias do milho, sugerem que além das características 
do equipamento, as características físicas dos ingredientes utilizados, em especial a densidade e granulometria, são importantes para determinar o tempo ideal de mistura das rações, com a necessidade de tempos específicos de mistura para cada DGM do milho.

Além disso, os resultados obtidos, para o milho pipoca e milho roxo moído, sugerem que o marcador ideal para granulometrias rotineiras pode não ser o mais adequado para indicar a qualidade de mistura de rações com ingredientes com granulometrias excessivamente diferentes das usuais. Esses resultados são corroborados por Axe (1995), que afirma que diferenças físicas entre ingredientes podem diminuir a qualidade da mistura. Esta similaridade com o milho da ração é um indicativo que o milho roxo moído e o milho pipoca, poderiam ser utilizados para avaliar a qualidade de mistura de rações com grande variação na granulometria dos ingredientes.

Além disso, trabalhos para a avaliação de diferentes graus de moagem do milho indicam que granulometrias excessivamente baixas ou altas tendem a piorar o desempenho (HEALY et al., 1994) e a digestibilidade da energia para suínos (WONDRA et al., 1995). Da mesma, forma pode reduzir o consumo de ração, de energia metabolizável e o ganho de peso para frangos de corte aos 42 dias de idade (RIBEIRO; MAGRO; PENZ JUNIOR, 2002). Para suínos o pior desempenho em granulometrias excessivamente finas pode estar associado ao maior desperdício de ração (MOREIRA et al., 2001) ou a irritação do trato digestório com desenvolvimento de queratose, enquanto as partículas de maior tamanho, por possuir menor área de superfície, em relação ao peso, tendem a diminuir a digestibilidade pelo menor contato entre enzimas digestivas e o alimento (HEALY et al., 1994). Para frangos pode estar associado à maior taxa de passagem e consequente menor tempo de digestão dos alimentos em granulometrias mais finas ou dificuldade de consumo de partículas grossas (RIBEIRO; MAGRO; PENZ JUNIOR, 2002).

Assim, os resultados obtidos neste trabalho indicam que a mistura menos eficiente, de rações com ingredientes com granulometrias excessivamente finas ou grossas, pode ser um fator importante para justificar o menor desempenho observado pelos autores nos extremos de granulometria.

Para os CV dos indicadores arroz, sorgo e microtracer F-Red, houve efeito quadrático $(\mathrm{P}<0,05)$ em relação ao tempo de acionamento utilizado e efeito linear $(\mathrm{P}<0,05)$ da granulometria do milho para o $\mathrm{CV}$ do sorgo e microtracers (Figura 2), o polinômio linear do arroz, embora não significativo $(\mathrm{P}>0,05)$, foi mantido em função da interação significativa. As derivações das equações obtidas permitiram estimar valores para tempo e DGM de 5,3 minutos e $-281 \mu \mathrm{m}$ para o arroz, 14,1 minutos e $630 \mu \mathrm{m}$ para o sorgo e 12,8 minutos e $569 \mu \mathrm{m}$ para o microtracer F-Red. 
Figura 2. Superfícies de respostas dos coeficientes de variação do sorgo, arroz e microtracers em rações fabricadas com diferentes tempos de mistura e diâmetros geométricos do milho.
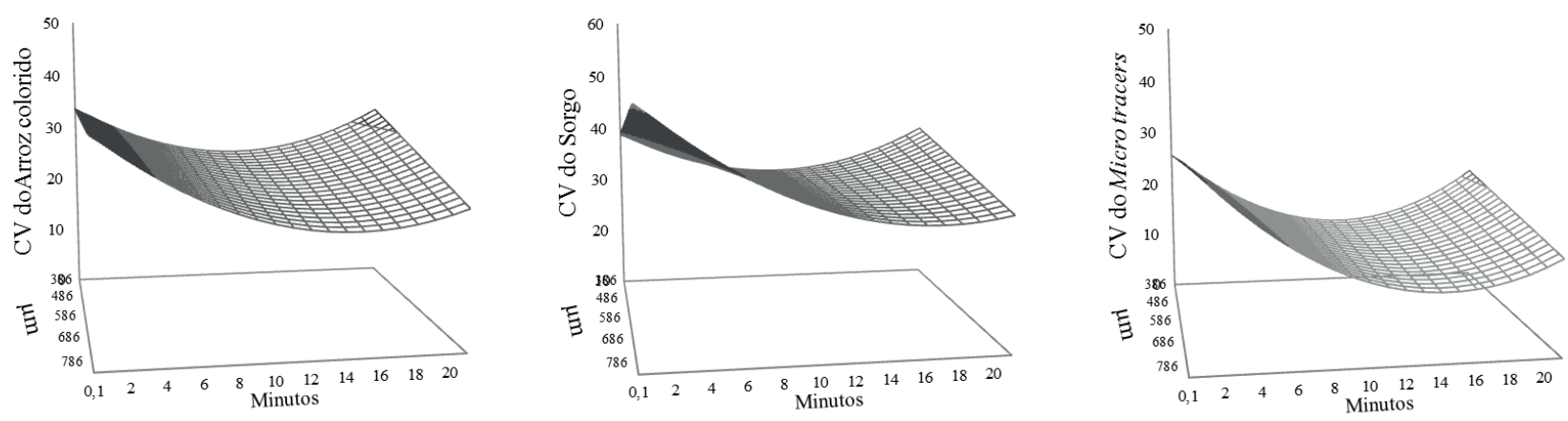

Sorgo: $\mathrm{CV}=28,1317-0,71354 \mathrm{~T}+0,0706912 \mathrm{~T}^{2}+0,0284376 \mathrm{D}-0,0020233 \mathrm{TD}$

Arroz colorido: $\mathrm{CV}=31,1556-1,17333 \mathrm{~T}+0,0763769 \mathrm{~T}^{2}+0,0068417 \mathrm{D}-0,0012865 \mathrm{TD}$

Microtracer F-red: CV=19,4610-1,93160 T+ 0,1052810 T² +0,0168824 D -0,0013236 TD.

Fonte: Elaboração dos autores.

No entanto, este método de derivação encontra o ponto em que a tangente se iguala ao zero, podendo ser encontrado valores demasiadamente fora do limite avaliado, como ocorreu para o arroz. Assumindo o limite de granulometria utilizado no experimento, no qual variou entre 385 e $839 \mu \mathrm{m}$ para o milho, foram obtidos tempos ideais de mistura entre 10,9 e 14,8 minutos para o arroz, 10,6 e 17,1 minutos para o sorgo, e 11,6 e 14,5 minutos para o microtracer F-red, respectivamente (Tabela 4). A interação entre tempo e granulometria e a diferença no tempo ideal de mistura para os diferentes indicadores, conforme o DGM do milho corrobora com a proposta de tempos específicos de mistura para diferentes granulometrias dos ingredientes utilizados.

Tabela 4. Tempos ideais de mistura, obtidos pelas derivações das equações em função do tempo, para as diferentes granulometrias do milho.

\begin{tabular}{lccccccc}
\hline \multirow{2}{*}{ DGM, $\mu \mathrm{m}$} & \multicolumn{7}{c}{ Indicadores } \\
\cline { 2 - 7 } & $\begin{array}{c}\text { Arroz } \\
\text { colorido }\end{array}$ & $\begin{array}{c}\text { Calcário } \\
\text { Grosso }\end{array}$ & Milheto & $\begin{array}{c}\text { Milho } \\
\text { Roxo }\end{array}$ & $\begin{array}{c}\text { Milho } \\
\text { Pipoca }\end{array}$ & Sorgo & $\begin{array}{c}\text { Microtracer } \\
\text { F-red }\end{array}$ \\
\hline 386 & 10,9 & 14,0 & 13,7 & 11,9 & 16,0 & 10,6 & 11,6 \\
513 & 12,0 & 14,0 & 13,7 & 10,2 & 12,3 & 12,4 & 12,4 \\
610 & 12,8 & 14,0 & 13,7 & 8,9 & 9,5 & 13,8 & 13,0 \\
731 & 13,8 & 14,0 & 13,7 & 7,4 & 6,1 & 15,5 & 13,8 \\
839 & 14,8 & 14,0 & 13,7 & 5,9 & 2,9 & 17,1 & 14,5 \\
\hline
\end{tabular}

Fonte: Elaboração dos autores.

Contudo, deve ser salientado que a movimentação da ração não é encerrada ao final do acionamento do misturador, o transporte por elevadores e transportadores, descarregamento, transporte rodoviário (da fábrica para as propriedades) assim como o armazenamento, as diferentes formas de arraçoamento e o próprio consumo animal, podem contribuir no processo de mistura e ou desmistura da ração. Por isso, as características similares dos indicadores propostos com os ingredientes da ração podem os tornar uma boa alternativa para a avaliação em nível de granjas da qualidade de mistura. 
Não houve interação $(\mathrm{P}>0,05)$ entre DGM do milho e tempo de acionamento do misturador e não houve efeito $(\mathrm{P}>0,05)$ do $\mathrm{DGM}$ do milho para o $\mathrm{CV}$ dos indicadores: calcário e milheto. Provavelmente, seus menores diâmetros de partícula e por consequência o maior número de unidades por $\mathrm{kg}$ de ração contribuíram com o resultado. A derivação das equações obtidas permitiu estimar os tempos de 14,0 e 13,7 minutos para o menor CV, respectivamente para o calcário e milheto (Figura 3).

O calcário e o milheto foram os ingredientes mais correlacionados com o método referência (Tabela 5). Provavelmente, o grande número de unidades destes indicadores por $\mathrm{kg}$ de ração, próximos ao número de partículas de microtracer F-Red (aproximadamente 1000 unidades/kg de ração), tenha contribuído para as maiores correlações com o método referência.
O calcário e o milheto foram os elementos que apresentaram a menor esfericidade e o menor peso por mil unidades e por consequência maior número de unidades por kg de ração, o que sugere que são as principais características a serem consideradas ao se propor um indicador alternativo.

Segundo Klein (2002), elementos como o cloreto de sódio, não são bons indicadores, pois o diâmetro geométrico médio das partículas do sal moído é grande, com resultados imprecisos para o $\mathrm{CV}$. Por outro lado, Groesbeck et al. (2007) verificaram que a redução das partículas de sal de $3000 \mu \mathrm{m}$ para 400 $\mu \mathrm{m}$ melhorou a avaliação da qualidade da mistura. Esta recomendação se assemelha aos resultados obtidos neste trabalho, no qual os elementos de menor esfericidade e por consequência maior quantidade de unidades por $\mathrm{kg}$ de ração, foram os mais correlacionados com o método referência.

Figura 3. Coeficientes de variação do calcário e milheto em rações fabricadas com diferentes tempos de mistura.

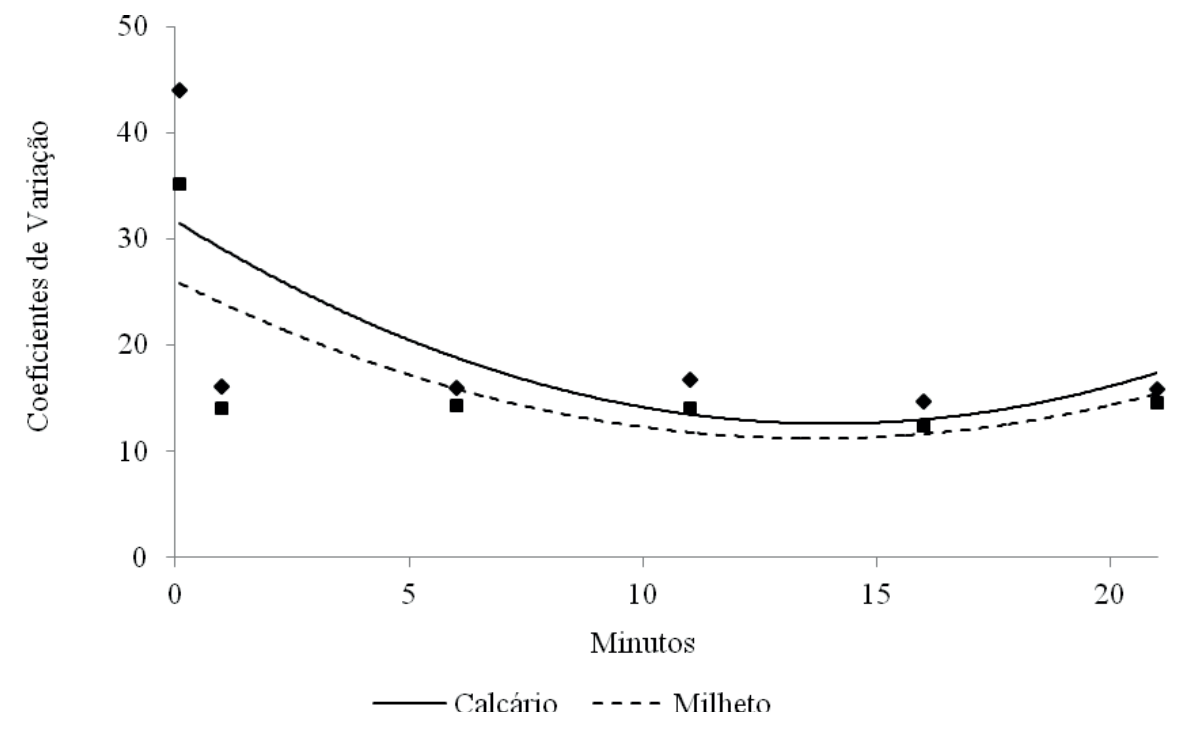

Calcário: $\mathrm{CV}=31,6930-2,72157 \mathrm{~T}+$ 0,0970223 T²; Milheto: $\mathrm{CV}=26,0166-2,15929 \mathrm{~T}+$ $0,0786770 \mathrm{~T}^{2}$.

Fonte: Elaboração dos autores. 
Tabela 5. Correlações de Pearson entre os coeficientes de variação dos indicadores avaliados para a qualidade de mistura.

\begin{tabular}{lllllll}
\hline & $\begin{array}{l}\text { Arroz } \\
\text { colorido }\end{array}$ & $\begin{array}{l}\text { Calcário } \\
\text { grosso }\end{array}$ & Milheto & Milho roxo & $\begin{array}{l}\text { Milho } \\
\text { pipoca }\end{array}$ & Sorgo \\
\hline Calcário grosso & $0,69^{*}$ & & & & & \\
Milheto & $0,64^{*}$ & $0,82^{*}$ & & & & \\
Milho roxo & 0,07 & 0,15 & 0,19 & & & \\
Milho pipoca & 0,01 & $-0,02$ & $-0,03$ & $-0,01$ & & \\
Sorgo & $0,60^{*}$ & $0,54^{*}$ & $0,47^{*}$ & 0,15 & $-0,10$ & \\
Microtracer F-red & $0,63^{*}$ & $0,70^{*}$ & $0,67^{*}$ & 0,12 & 0,08 & $0,57^{*}$ \\
\hline
\end{tabular}

$* \mathrm{P}<0,01$.

Fonte: Elaboração dos autores.

Entretanto, deve ser destacado que a quantificação dos teores de cloreto de sódio é realizada por titulação, enquanto que neste experimento os indicadores foram avaliados por contagem. Mesmo com as diferenças, nas técnicas de mensuração, houve similaridade nos resultados, pois os mais correlacionados com o método referência foram os de menor diâmetro.

Clark, Behnke e Poole (2007) recomendam a utilização de ingredientes de baixa inclusão e que não sejam componentes dos alimentos usuais da ração como, por exemplo: os antibióticos ou aminoácidos livres. Entretanto, esta metodologia pode elevar o custo e o tempo necessário para a avaliação dos tempos ideais de mistura.

Neste sentido, como não há contribuição dos componentes da ração para a quantificação dos indicadores, não há demanda de grande aparato laboratorial para a análise (pesagem, peneiramento e a avaliação visual permitem sua quantificação), o que reduz o tempo e o custo de avaliação, o uso de macro-ingredientes, se mostrou promissor, como ferramenta complementar, para determinação dos tempos ideais de mistura.

Entre os indicadores avaliados houve destaque para o milheto e o calcário, os quais apresentaram as maiores correlações com o método referência, provavelmente pelo seu pequeno diâmetro e grande número de unidades por $\mathrm{kg}$ de ração. Entretanto, outros estudos devem ser realizados, com rações com composições centesimais diferentes e em misturadores do tipo horizontal e ou vertical.

\section{Conclusões}

A granulometria do milho alterou o tempo necessário para reduzir o coeficiente de variação dos indicadores, arroz colorido, milho roxo moído, milho pipoca, sorgo e microtracer F-red. $\mathrm{O}$ uso de macro-ingredientes, como indicadores alternativos, se mostrou promissor como ferramenta complementar para determinação dos tempos ideais de mistura, com destaque para o milheto e o calcário, os quais apresentaram as maiores correlações com o método referência.

\section{Referências}

AMERICAN SOCIETY OF AMERICAN SOCIETY OF AGRICULTURAL ENGINEERS - ASAE. S303.3. Test procedure for solids-mixing equipment for animal feeds. In: ASAE standards 1998: standards Engineering Practices Data, St. Joseph, 1998. p. 132-136.

AXE, D. E. Factors affecting uniformity of a mix. Animal Feed Science and Technology, Amsterdam, v. 53, n. 2, p. 211-220, 1995.

CLARK, P. M.; BEHNKE, K. C.; POOLE D. R. Effects of marker selection and mix time on the coefficient of variation (mix uniformity) of broiler feed. Journal of Applied Poultry Research, Champaign, v. 16, n. 3, p. 464-470, 2007. 
GROESBECK, C. N.; GOODBAND, R. D.; TOKACH, M. D.; DRITZ, S. S.; NELSSEN, J. L.; DEROUCHEY, J. M. Diet mixing time affects nursery pig performance. Journal of Animal Science, Champaign, v. 85, n. 7, p. 1793-1798, 2007.

HANDERSON, S. M.; PERRY, R. L. Agricultural process engineering. New York: John Wiley and Sons, 1955. $402 \mathrm{p}$.

HEALY, B. J.; HANCOCK, J. D.; KENNEDY, G. A.; BRAMEL-COX, P. J.; BEHNKE, K. C.; HINES, R. H. Optimum particle size of corn and hard and soft sorghum for nursery pigs. Journal of Animal Science, Champaign, v. 72, n. 9, p. 2227-2236, set. 1994.

JOHNSTON, S. L.; SOUTHERN, L. L. The effect of varying mix uniformity (simulated) of phytase on growth performance, mineral retention, and bone mineralization in chicks. Poultry Science, Champaign, v. 79, n. 10, p. 1485-1490, 2000.

KLEIN, A. A. Pontos críticos no processo de fabricação de rações. In: SIMPÓSIO GOIANO DE AVICULTURA, 2002, Goiânia. Anais... Goiânia: Associação Goiana de Avicultura, UFG, 2002, p. 57-78.

MAGALHÃES, A. C.; COUTO, S. M.; QUEIROZ, D. M.; ANDRADE, E. T. Dimensões principais, massa e volume unitários, esfericidade e ângulo de repouso de frutos de café. Revista Brasileira de Produtos Agroindustriais, Campina Grande, v. 2, n. 2, p. 39-56, 2000.

McCOY, R. A. K. C.; BEHNKE, K. C.; HANCOCK, J. D.; MCELLHINEY, R. R. Effect of mixing uniformity on broiler chick performance. Poultry Science, Champaign, v. 73, n. 3, p. 443-451, 1994.

MELLO, R. C. A.; PUPA, J. M. R.; HANNAS, M. I. Mistura de rações: um ponto chave no sistema de produção animal. Revista Allnutri, Viçosa, v. 3, n. 1, p. $1-4,2003$.

MICROTRACERS. Instructions for microtracer (tm) "Mason Jar" TEST for feeds formulated with microtracers F. San Francisco: Microtracers, 1999. p. 1-3.
MOREIRA, I.; OLIVEIRA, G. C.; FURLAN, A. C.; PATRICIO, V. I.; MARCOS JUNIOR, M. Utilização da farinha pré-gelatinizada de milho na alimentação de leitões na fase de creche. Digestibilidade e desempenho. Revista Brasileira de Zootecnia, Viçosa, v. 30, n. 2, p. 440-448, 2001.

MOREIRA, S. M. C.; CHAVES, M. A.; OLIVEIRA, L. M. Comparação da eficiência de líquidos na determinação da massa específica aparente de grãos agrícolas. Revista Brasileira de Armazenamento, Campina Grande, v. 9, n. 1, p. 22-24, 1985.

RIBEIRO, A. M. L.; MAGRO, N.; PENZ JÚNIOR, A. M. Granulometria do milho em rações de crescimento de frangos de corte e seu efeito no desempenho e metabolismo. Revista Brasileira de Ciencia Avícola, Campinas, v. 4, n. 1, p. 1-7, 2002.

ROSTAGNO, H. S.; ALBINO, L. F. T.; DONZELE, J. L.; GOMES, P. C.; OLIVEIRA, R. F.; LOPES, D. C.; FERREIRA, A. S.; BARRETO, S. L. T.; EUCLIDES, R. F. Tabelas brasileiras para aves e suínos: composição de alimentos e exigências nutricionais. 3. ed. Viçosa, MG: UFV, 2011. 252 p.

TRAYLOR, S. L.; HANCOCK, J. D.; BEHNKE, K. C.; STARK, C. R.; HINES. R. H. Uniformity of mixed diets affects growth performance in nursery and finishing pigs. Journal of Animal Science, Champaign, v. 72, n. 2, p. 171-175, 1994.

WONDRA, K. L.; HANCOCK, J. D.; BEHNKE, K. C.; HINES, R. H.; STARK, C. R. Effects of particle size and pelleting on growth performance, nutrient digestibility, and stomach morphology in finishing pigs. Journal of Animal Science, Champaign, v. 73, n. 3, p. 757-763, 1995.

ZANI, A. Projeta apenas recuperação das perdas acumuladas no ano passado. Setor de alimentação animal. São Paulo: SINDIRAÇÕES, 2013. 8 p. 
